\title{
Demonstration of resistance to satyrization behavior in Aedes aegypti from La Réunion island
}

\author{
Hamidou Maïga ${ }^{1,2, *}$, Jérémie R. L. Gilles ${ }^{1}$, Rosemary Susan Lees ${ }^{3}$, Hanano Yamada ${ }^{1}$, and Jérémy Bouyer ${ }^{1, *}$ \\ ${ }^{1}$ Insect Pest Control Laboratory, Joint FAO/IAEA Division of Nuclear Techniques in Food and Agriculture, International Atomic Energy \\ Agency, PO Box 100, 1400 Vienna, Austria \\ 2 Institut de Recherche en Sciences de la Santé/Direction Régionale de l'Ouest, 01 BP 545 Bobo 01, Bobo-Dioulasso, Burkina Faso \\ ${ }^{3}$ Department of Vector Biology, Liverpool School of Tropical Medicine, Pembroke Place, Liverpool, L3 5QA, United Kingdom
}

Received 16 February 2020, Accepted 21 March 2020, Published online 7 April 2020

\begin{abstract}
Aedes aegypti and Aedes albopictus are competent vectors of arboviruses such as dengue and chikungunya viruses which co-exist in some areas, including La Réunion island, Indian Ocean. A type of reproductive interference called satyrization has been described in sympatric species where dominant species mating fails to produce hybrids and thus reduces the fitness and tends to control the spread of the other species. Here, we investigated satyrization in laboratory experiments to provide insights on the potential impact on Ae. aegypti of a control campaign including a sterile insect technique component against Ae. albopictus. Different mating crosses were used to test sympatric, conspecificinterspecific and allopatric effects of irradiated and non-irradiated male Ae. albopictus on female Ae. aegypti, including in a situation of skewed male ratio. Our results suggest that there was only a low level of satyrization between sympatric populations of Ae. aegypti and Ae. albopictus colonized from La Réunion island. A male Ae. albopictus to female Ae. aegypti ratio of 3:1 did not increase the level of satyrization. Female Ae. aegypti previously mated to male Ae. albopictus were not prevented from being inseminated by conspecific males. A satyrization effect was not seen between allopatric Ae. albopictus and Ae. aegypti strains from La Réunion Island either. The tested Ae. aegypti strain from La Réunion island has therefore developed full resistance to satyrization and so releasing sterile male Ae. albopictus may not suppress Ae. aegypti populations if an overflooding of irradiated male Ae. albopictus leads to similar results. The management strategy of two competent species in a sympatric area is discussed.
\end{abstract}

Key words: Aedes albopictus, Satyrization, Sterile insect technique, Mating.

Résumé - Démonstration de la résistance au comportement de satyrisation chez Aedes aegypti de l'île de La Réunion. Aedes aegypti et Aedes albopictus sont des vecteurs compétents d'arbovirus, tels que les virus de la dengue et du chikungunya, qui coexistent dans certaines régions, y compris à La Réunion, dans l'Océan Indien. Une interférence sur la reproduction appelée satyrisation a été décrite chez les espèces sympatriques où l'accouplement d'une espèce dominante ne produit pas d'hybrides et donc réduit le succès reproducteur et tend à contrôler la propagation des autres espèces. Ici, nous avons étudié la satyrisation dans des expériences de laboratoire pour fournir des informations sur l'impact potentiel sur Ae. aegypti d'une campagne de lutte contre Ae. albopictus basée sur la technique de l'insecte stérile. Différents croisements d'accouplements ont été utilisés pour tester les effets sympatriques, conspécifiques-interspécifiques et allopatriques de mâles Ae. albopictus irradiés et non irradiés sur les femelles Ae. aegypti, y compris dans une situation de ratio masculin asymétrique. Nos résultats suggèrent qu'il n'y avait qu'un faible niveau de satyrisation entre les populations sympatriques d'Ae. aegypti et Ae. albopictus colonisées à La Réunion. Un rapport mâles Ae. albopictus sur femelles Ae. aegypti de 3 contre 1 n'a pas augmenté le niveau de satyrisation. Les femelles Ae. aegypti déjà accouplées à un mâle Ae. albopictus n'ont pas été empêchées d'être inséminées par des mâles conspécifiques. Aucun effet de satyrisation n'a été observé entre les souches allopatriques d'Ae. albopictus et d'Ae. aegypti de La Réunion. La souche testée d'Ae. aegypti de La Réunion a donc développé une résistance totale à la satyrisation et en conséquence la libération de mâles stériles d'Ae. albopictus peut ne pas supprimer les populations d'Ae. aegypti si des lâchers massifs de mâles irradiés d'Ae. albopictus conduisent à des résultats similaires. La stratégie de gestion de deux espèces compétentes dans une zone sympatrique est discutée.

*Corresponding authors: h.maiga@iaea.org; j. bouyer@iaea.org

This is an Open Access article distributed under the terms of the Creative Commons Attribution License (https://creativecommons.org/licenses/by/4.0), which permits unrestricted use, distribution, and reproduction in any medium, provided the original work is properly cited. 


\section{Introduction}

Aedes albopictus (Skuse), the Asian tiger mosquito, has been invasive in many parts of the world since the 1980s [10]. Aedes aegypti (Linnaeus), also called the yellow fever mosquito and originating from Africa, is a highly invasive, medically important mosquito species that has received a considerable increase in attention after being linked to the Zika outbreak in Brazil in 2015 [19]. Both species transmit several arboviral diseases including dengue, chikungunya, yellow fever, and Zika [37]. Dengue alone is estimated to infect 390 million people per year, causing 96 million cases with clinical manifestations [11]. Aedes albopictus (Skuse) and Ae. aegypti are the most important vectors of the epidemic forms of dengue and chikungunya viruses to humans [36, 44]. Aedes albopictus is also responsible for the major chikungunya outbreak in the islands of the South-West Indian Ocean, including La Réunion island (an overseas department of France) between 2005 and 2007 [20] and for the more recent dengue outbreaks according to the local health authority (Agence Régionale de Santé Océan Indien) and the regional office of Santé publique France on La Réunion island [50].

The heavy reliance on insecticides to control adult Aedes mosquitoes, especially during disease outbreaks, has led to the emergence of widespread resistance to these chemicals, making traditional control strategies insufficient to achieve adequate reduction of vector populations. The use of insecticides is also inefficient against these container-breeding mosquito species with disseminated larval habitats. Therefore, complementary vector control methods are needed to enhance existing efforts [49]. Amongst those being advocated is the sterile insect technique (SIT) [12], a species-specific and environmentallyfriendly pest population control method that relies on maintaining a continuous production and repeated release of overflooding numbers of sterile males [35] that can outcompete their wild counterparts within the target area [21], and induce sterility in wild females. A feasibility assessment of an areawide integrated pest management (AW-IPM) program with an SIT component is ongoing on La Réunion island, where Ae. albopictus co-occurs with Ae. aegypti. The first releases of sterile male Ae. albopictus mosquitoes to study their behavior in urban areas were recently authorized by a prefectural order to the Institute for Research for Development (IRD) [2]. To successfully and cost-effectively apply the SIT in an area, it is recommended to target one species at a time [1, 21]. For example, where Ae. aegypti and Ae. albopictus are sympatric and are both competent vectors of human viruses, one would consider the best approach to guarantee successful suppression or elimination of both species. The best scenario would be the release of one species which was able to also readily mate with and induce sterility in both species [27]. This phenomenon is known as reproductive interference or satyrization, in which males of one species mate with and sterilize females of another species, and in this way contribute to its displacement from the shared area [45].

Satyrization was proposed as a possible mechanism for the displacement of Ae. aegypti by Ae. albopictus in Florida in the late 1980s [43, 48]. Satyrization and other factors such as larval competition, climate, and socioeconomic factors have influenced the distribution dynamics of the two species worldwide. A rapid decline in Ae. aegypti in the south eastern USA and Bermuda, for example, was associated with the invasions of Ae. albopictus [33, 38, 43]. The same mechanism was suggested to explain the spread of invasive Ae. albopictus in Athens, Greece and the reduction in distribution of the native Ae. cretinus [25]. Displacement of Ae. albopictus by Ae. aegypti has also occurred in certain tropical cities and regions in Asia including Bangkok, Kuala Lumpur, Manilla, and southern Taiwan, and more recently in the Colombian port of Leticia (reviewed in [39]). However, it is suggested that resistance to satyrization may evolve more rapidly in female Ae. aegypti populations sympatric to Ae. albopictus than in females from allopatric populations [6, 40]. Bagny et al. [3] reported a progressive decrease in Ae. aegypti distribution on La Réunion island since the 1900s where it was mainly found in rock holes with Ae. albopictus in ravines located on the driest west coast of the island and was absent from artificial containers. The same study suggested that the dwindling Ae. aegypti densities observed during the 1950 s was due to ecological factors including a competitive interaction between Ae. aegypti and Ae. albopictus combined with vector control campaigns during these years. However, to the best of our knowledge, the competitive interaction between La Réunion island strains has not yet been investigated.

With both Ae. aegypti and Ae. albopictus being competent vectors of dengue and several other arboviruses including chikungunya and Zika viruses [16, 38, 44], it is important to investigate whether or not the release of irradiated Ae. albopictus males would affect Ae. aegypti populations [17], in the framework of the SIT project on La Réunion island. Our satyrization experiments provide insights on the potential impact on Ae. aegypti of a control campaign against Ae. albopictus on La Réunion island. Different mating crosses were used to test sympatric, conspecific-interspecific, and allopatric effects of male Ae. albopictus on female Ae. aegypti including in a situation of skewed male ratio. A preprint of this work was published on February 12, 2020 [41].

\section{Materials and methods}

\section{Mosquito strains, rearing, and irradiation conditions}

The Ae. aegypti strain used in this study originated from field collections on La Réunion island. The strain was colonized in the laboratory by the Institute of Research for Development (IRD) for five generations (F5) before being transferred to the Insect Pest Control Laboratory (IPCL) of the joint FAO/IAEA division of Nuclear Sciences and Applications in 2016. The Ae. albopictus La Réunion island strain was maintained at the IPCL from 2009 for several generations before these experiments were performed in 2016. In order to perform different mating crosses, Ae. albopictus strains from China (Guangzhou wild type strain, provided by Wolbaki since 2015) and Italy (Rimini strain, provided by Centro Agricoltura Ambiente (CAA) since 2012) were maintained in parallel.

All the strains were reared in $30 \times 40 \times 10 \mathrm{~cm}$ trays at a density of 1 first instar larvae (L1) per mL under controlled 
temperature, humidity, and lighting conditions $\left(T=26 \pm 2{ }^{\circ} \mathrm{C}\right.$, $70 \pm 10 \mathrm{RH} \%, 12: 12 \mathrm{~h}$ light: dark, including $1 \mathrm{~h}$ dawn and $1 \mathrm{~h}$ dusk). Larvae were fed with IAEA larval diet following the protocol described in the Guidelines for routine rearing [22]. Pupae were collected and separated using a glass plate sorter [23].

Male pupae of all strains were irradiated between $36 \mathrm{~h}$ and 44 h of age with 40 Gy using a Gamma Cell 220 (Nordion Ltd., Kanata, ON, Canada) emitting a dose rate of $90 \mathrm{~Gy} / \mathrm{min}$.

\section{Experiment 1. Sympatric cross-mating between Ae. aegypti and Ae. albopictus strains from La Réunion island}

Male Ae. aegypti and Ae. albopictus were irradiated and crossed with female Ae. albopictus and Ae. aegypti, respectively. Non-irradiated males of each species were also crossed with female mosquitoes of the other species. Non-irradiated male Ae. aegypti and Ae. albopictus were also crossed with virgin female Ae. aegypti and Ae. albopictus, respectively, and used as controls.

Three replicates were performed for each cross with 50 males and 50 females transferred to $15 \times 15 \times 15 \mathrm{~cm}$ cages (MegaView Science Co. Ltd., Taiwan) when they were three days old for a period of seven days to ensure enough time was allowed for mating. Females were offered a defibrinated porcine bloodmeal using sausage casings (Grade Specification: $326 \mathrm{NC}$, EDICAS Co., Ltd.) for $1 \mathrm{~h}(2 \times 30 \mathrm{~min}$ with $10 \mathrm{~min}$ reheating of the blood sausage in between feedings) on two consecutive days when they were 5-6 days old. Each of the females was transferred to an individual drosophila tube containing a cone of seed germination paper (Grade 6, Size: $580 \times 580 \mathrm{~mm}$, weight: $145 \mathrm{~g} / \mathrm{m}^{2}$, Sartorius Stedim Biotech) and $10 \mathrm{~mL}$ of water. Females were given three days to lay eggs and then dissected to determine their insemination status under a stereomicroscope $(40 \times$ magnification). Before dissection, females were kept in labelled $50 \mathrm{~mL}$ Falcon tubes (VWR International $\mathrm{GmbH}$, Darmstadt, Germany) in a refrigerator at $4{ }^{\circ} \mathrm{C}$, and samples held in a cold box containing ice to avoid desiccation while other samples were being dissected.

Virgin Ae. aegypti and Ae. albopictus females of the same batch were offered blood meals and were also placed in individual egging tubes to assess their egging capacity.

All eggs were collected, dried for seven days in the laboratory and allowed to hatch for $20 \mathrm{~h}$ with a hatching solution made of $0.25 \mathrm{~g}$ of CM 0001 Nutrient Broth (Oxoid, Hampshire, UK) and $0.05 \mathrm{~g}$ of yeast diluted in $0.7 \mathrm{~L}$ of deionized water [51].

The number of female mosquitoes still alive after seven days together with males was recorded for each replicate, and the survival rate was compared with survival in the conspecific Ae. aegypti mating control.

\section{Experiment 2. Effect of male Ae. albopictus density (ratio) on female Ae. aegypti mating success}

To assess whether an increase in male to female ratio would favour satyrization, female Ae. aegypti mosquitoes from La Réunion island were allowed to mate with male
Ae. albopictus in a male: female ratio of 3:1 corresponding to 75 male Ae. albopictus and 25 female Ae. aegypti. Three days-old males and females were held in $15 \times 15 \times 15 \mathrm{~cm}$ BugDorm cages (MegaView Science Co. Ltd., Taiwan) for seven days. The crosses were performed using irradiated males (four replicates) and non-irradiated males (seven replicates). Females were then dissected to check their insemination status, as described above. This experiment was carried out in parallel with Experiment 1 and results of mating crosses could be compared to the insemination rates obtained with a 1:1 male: female ratio (50 males $\times 50$ females).

\section{Experiment 3. Pre-exposure effect on mating success (interspecific-conspecific treatment)}

To assess whether female Ae. aegypti that were pre-exposed to male Ae. albopictus could re-mate with their conspecific male Ae. aegypti, 4-5 days-old female Ae. aegypti (La Réunion island strain) were pre-exposed to irradiated and non-irradiated male Ae. albopictus (La Réunion island strain). Females were removed after seven days and were offered to irradiated and non-irradiated male Ae. aegypti (La Réunion island strain) for another seven days. Females were then blood fed for two consecutive days and eggs collected before females were dissected under a microscope to determine insemination status. We hypothesized that if female Ae. aegypti were inseminated by non-irradiated Ae. albopictus males, they would not be able to lay fertile eggs even if they had a blood meal.

Fifty adults were included in each cross in a 1:1 male: female ratio in Bugdorm cages $(15 \times 15 \times 15 \mathrm{~cm})$.

An experimental design was used which ensured that female Ae. aegypti that were pre-exposed to male Ae. albopictus had not been inseminated (Table 1), and female insemination and egg hatch rates were observed.

\section{Experiment 4. Effect of geographic origin on mating success (allopatric crosses)}

Since we observed resistance to satyrization against Ae. albopictus in the sympatric cross experiment, we explored the allopatric effect to better understand the mating behavior of the La Réunion island strain of Ae. aegypti. Several crosses were performed using irradiated and non-irradiated males of the Ae. albopictus strains from La Réunion island, China (Guangzhou wild type strain) and Italy (Rimini strain).

Female Ae. aegypti mosquitoes from La Réunion island were crossed for seven days with non-irradiated or irradiated male Ae. albopictus mosquitoes from either La Réunion island, China or Italy, in 14 combinations. In each interspecific treatment (Ae. aegypti female $\times$ Ae. albopictus male), 30 males and 30 females, three-days old, from each strain were housed in $15 \times 15 \times 15 \mathrm{~cm}$ BugDorm cages. In addition, conspecific control crosses (Ae. albopictus female $\times$ Ae. albopictus male, and $A$ e. aegypti female $\times A e$. aegypti male, La Réunion island strain) were carried out using the same number of males and females for each strain. The number of females that were successfully inseminated was recorded (as described for Experiment 1) and results were compared to the interspecific 
Table 1. Experimental design of the interspecific-conspecific treatments.

\begin{tabular}{lccccccc}
\hline Cross & Status & $\begin{array}{c}\text { Number of } \\
\text { replicts }\end{array}$ & Male & Female & $\begin{array}{c}\text { Irradiated } \\
\text { males }\end{array}$ & $\begin{array}{c}\text { Fecundity and } \\
\text { fertility checks }\end{array}$ & $\begin{array}{c}\text { Dissection for } \\
\text { insemination status check }\end{array}$ \\
\hline C1 & Control & 5 & AEG & AEG & Yes & No & Yes \\
C2 & Control & 4 & AEG & AEG & No & No & Yes \\
C3 & Control & 4 & ALBO & ALBO & Yes & No & Yes \\
C4 & Control & 4 & ALBO & ALBO & No & No & Yes \\
C5 & Pre-exposed & 4 & ALBO & AEG & Yes & No & Yes \\
C6 & Pre-exposed & 4 & ALBO & AEG & No & No & Yes \\
C7 & Control & 5 & AEG & AEG & No & Yes & Yes \\
C8 & Pre-exposed & 5 & ALBO & AEG & No & Yes & Yes \\
\hline
\end{tabular}

AEG and ALBO stand for Ae. aegypti and Ae. albopictus, respectively; C for cross, and numbers 1-8 for cross identity.

crosses and to the conspecific control crosses. Egg hatch was performed as described for Experiment 1 for batches that were collected from females mated with non-irradiated males.

\section{Statistical analysis}

Statistical analyses were performed and graphs drawn using RStudio Team [46]. The proportion of inseminated females was calculated as the number with at least one spermathecae filled with sperm divided by the number of dissected females. The proportion of egg hatch was calculated from the number of hatched eggs divided by the total number of eggs laid per individual female (Experiment 1) or per group of females in a cage (Experiments 3 and 4). The proportions were transformed following the Freeman-Tukey method (arcsine transformed data). An analysis of variance (ANOVA) was then performed followed by a Tukey multiple comparison to compare means of each crossed pair. A paired $t$-test was performed to compare egg hatch between pre-exposed and non-exposed females (pre-exposure effect on mating success experiment).

The survival rate (Experiment 1) was analyzed using a generalized binomial linear mixed-effects model fit by maximum likelihood (Laplace approximation) with logit link with the survival rate, defined as dependent variable, and type of mating pairs (cross) as fixed effects, and replicates as a random effect. The best model was selected based on the lowest corrected Akaike information criterion (AICc), and the significance of fixed effects was tested using the likelihood ratio test [14, 29].

The statistical significance for all experiments was determined at $\alpha=0.05$ level.

\section{Results}

\section{Experiment 1. Sympatric cross-mating between Ae. aegypti and Ae. albopictus strains from La Réunion island}

There was no insemination between sympatric species from La Réunion island strains either when non-irradiated or irradiated male Ae. aegypti or Ae. albopictus were caged with female Ae. albopictus $(n=82$, mean $( \pm \mathrm{SE})=0 \pm 0 \%$ and $n=72$, mean $( \pm \mathrm{SE})=0 \pm 0 \%)$ or Ae. aegypti $(n=28$, mean $( \pm \mathrm{SE})=0 \pm 0 \%$ and $n=41$, mean $( \pm \mathrm{SE})=0 \pm 0 \%)$, respectively, compared to the conspecific mating controls (Ae. albopictus: $n=58$, mean
$( \pm \mathrm{SE})=99.2 \pm 0.01 \% ;$ Ae. aegypti: $n=71$, mean $( \pm \mathrm{SE})=100 \pm 0 \%)\left(\right.$ Fig. $\left.1, F_{(5,12)}=735.6, p<0.0001\right) . \mathrm{A}$ Tukey multiple comparison of means did not show any difference in insemination rate regardless of the cross type between irradiated or non-irradiated males of Ae. albopictus or Ae. aegypti and non-irradiated females of Ae. aegypti and Ae. albopictus, respectively $(p>0.05)$.

Regardless of the male status (irradiated or non-irradiated) the females exposed to interspecific mating, and virgin females, laid a lower number of eggs per female than the females of the conspecific mating control crosses (Interspecific: (C3-6): 146 eggs $(n=223)$; virgin females (Faeg-Falbo): 92 eggs $(n=77)$, controls $(\mathrm{C} 1-2)$ : 2047 eggs $(n=129))$. None of the eggs from interspecific mating crosses and virgin females hatched compared to the controls (Fig. 2, $F_{(7,46)}=84.73$, $p<0.0001)$.

Interspecific mating was more detrimental to female Ae. aegypti survival than to Ae. albopictus (Table 2, $p<0.0001)$.

\section{Experiment 2. Effect of male Ae. albopictus density (ratio) on female Ae. aegypti mating success}

Increasing the ratio of male: female to $3: 1$ in favor of male Ae. albopictus did not significantly increase the satyrization effect on female Ae. aegypti (La Réunion island) judged by insemination rates for both non-irradiated ( $t$-test, ratio 1:1: mean $( \pm \mathrm{SE})=0 \pm 0 \%, n=28$, ratio $3: 1$ : mean $( \pm \mathrm{SE})=2 \pm 0.1 \%$, $n=169, p=0.26)$ and irradiated ( $t$-test, ratio 1:1: mean $( \pm \mathrm{SE})=0 \pm 0 \%, n=41$, ratio $3: 1$ : mean $( \pm \mathrm{SE})=0 \pm 0 \%$, $n=101, p=1)$ male Ae. albopictus. All these crosses had negligible insemination rates compared to conspecific mating (controls) $\left(F_{(7,21)}=272.8, p<0.0001\right)$.

\section{Experiment 3. Pre-exposure effect on mating success}

When female Ae. aegypti were pre-exposed to nonirradiated or irradiated male Ae. albopictus, there was no insemination (Fig. 3, C5: mean $( \pm \mathrm{SE})=0 \pm 0 \%, n=90$, and C6: mean $( \pm \mathrm{SE})=0 \pm 0 \%, n=121)$, whereas groups of females that were pre-exposed to male Ae. albopictus were inseminated by their male Ae. aegypti conspecifics (Fig. 3, C7: mean $( \pm \mathrm{SE})=$ $100 \pm 0 \%, n=106$, and C8: mean $( \pm \mathrm{SE})=95.99 \pm 0 \%$, 


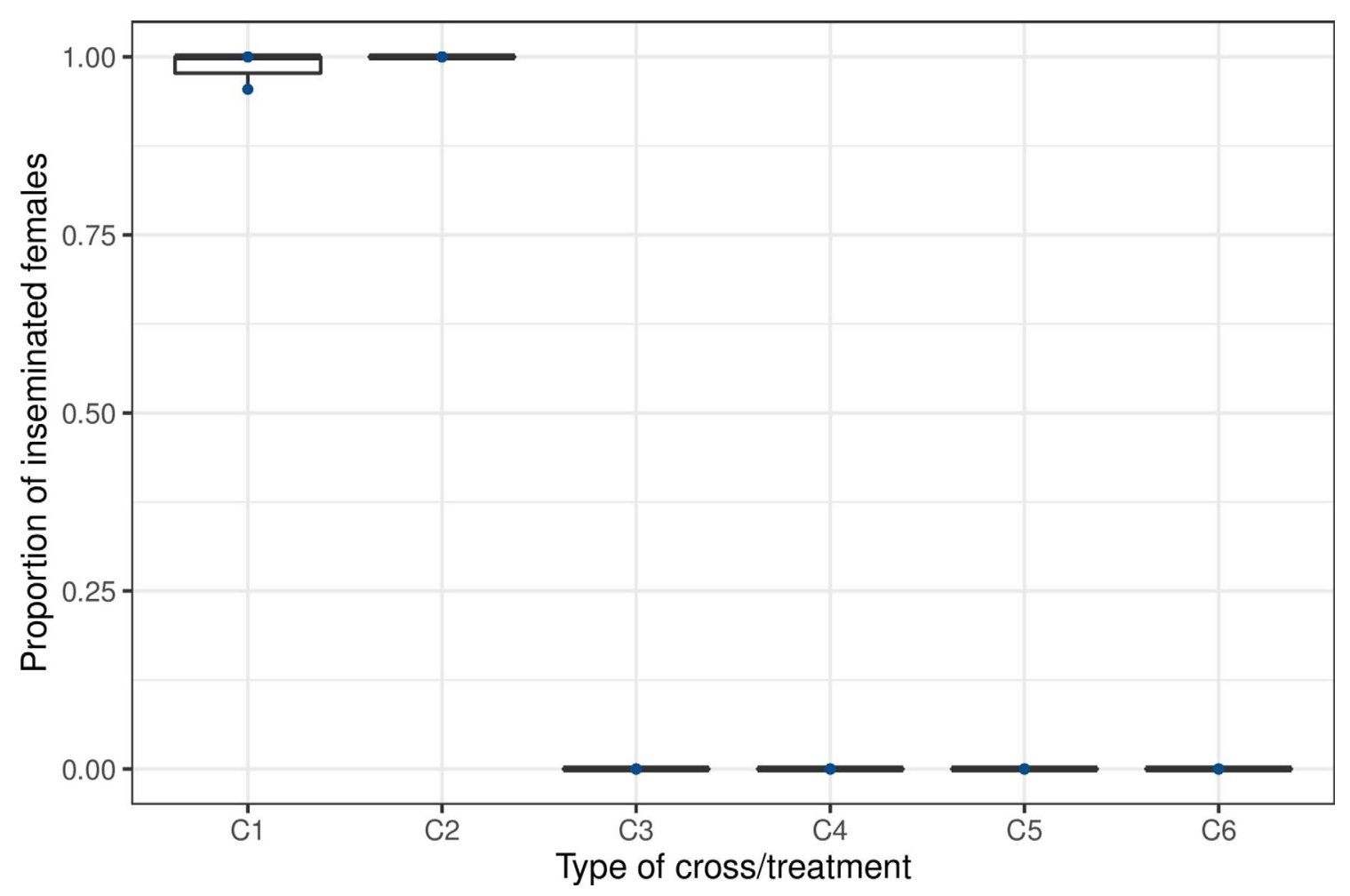

Figure 1. Sympatric cross-mating between Ae. aegypti and Ae. albopictus strains from La Réunion island. C denotes the cross, and numbers (1-6) are related to the cross identity. The number of dissected females $(n)$ per cross were $\mathrm{C} 1=$ control Ae. albopictus (non-irradiated), $n=58$; $\mathrm{C} 2=$ control Ae. aegypti (non-irradiated), $n=71 ; \mathrm{C} 3=$ male Ae. albopictus (non-irradiated) $\times$ female Ae. aegypti, $n=28 ; \mathrm{C} 4=\mathrm{male}$ Ae. albopictus (irradiated) $\times$ female Ae. aegypti, $n=41 ; \mathrm{C} 5=$ male Ae. aegypti (non-irradiated) $\times$ female Ae. albopictus, $n=82 ; \mathrm{C} 6=$ male Ae. aegypti (irradiated) $\times$ female Ae. albopictus, $n=72$.

$n=146)$. No difference in female insemination rates was observed between pre-exposed and non-exposed females $(p=0.27)$ or between pre-exposed females and controls (Fig. 3, C1-3 vs. C7-8) $(p>0.05)$.

Female Ae. aegypti that were pre-exposed to male Ae. albopictus successfully laid fertile eggs when they then mated with their male Ae. aegypti conspecifics, and mean egg hatch was lower but not significantly different between the non-exposed $($ mean $( \pm \mathrm{SE})=95.99 \pm 0.008 \%)$ and pre-exposed females $($ mean $( \pm \mathrm{SE})=91.61 \pm 0.02 \%)(t$-test: $t=2.0576$, $d f=4, p=0.1)$.

\section{Experiment 4. Effect of geographic origin on mating success (allopatric crosses)}

A greater female insemination rate was observed when conspecific mating was compared to the interspecific mating groups, regardless of the male origin or irradiation status (Table 3, $\left.F_{(13,23)}=229.1, p<0.0001\right)$. Neither geographic origin nor irradiation status of male Ae. albopictus had an impact on female Ae. aegypti insemination rates, confirming the conclusion that allopatric and sympatric effects were similar when the La Réunion island strain of Ae. aegypti was used in these experiments (Table 3, $p>0.05$ ).

We observed a significantly lower egg hatch in interspecific than conspecific crosses, irrespective of the male origin (Table 3 , $\left.F_{(13,23)}=182.9, p<0.0001\right)$.

\section{Discussion}

The objective of this study was to assess the level of reproductive interference between male Ae. albopictus and female Ae. aegypti, and to discuss the management strategy for the two vector species in a sympatric area. In laboratory conditions, almost no satyrization effect was observed between sympatric populations of Ae. aegypti and Ae. albopictus colonized from La Réunion island, even when the male Ae. albopictus to female Ae aegypti ratio was increased to 3:1. Female Ae. aegypti previously exposed to male Ae. albopictus were not prevented from being inseminated by conspecific male Ae. aegypti. Satyrization did not occur between allopatric Ae. albopictus and Ae. aegypti strains from La Réunion island either. An Ae. aegypti strain from La Réunion island has therefore developed full resistance to satyrization (anti-satyrization behavior).

Many reasons could explain the observed low level of satyrization. Bargielowki et al. [6,7] have previously described sympatric field populations that have co-existed long enough to evolve resistance to cross-mating and showed that Ae. aegypti females allopatric to Ae. albopictus are more susceptible to interspecific insemination by Ae. albopictus [40]. Cage experiments and field observations indicate that Ae. albopictus males are capable of satyrizing females of other species of the Stegomyia subgenus, potentially leading to competitive displacement, and even extinction, especially of endemic species 


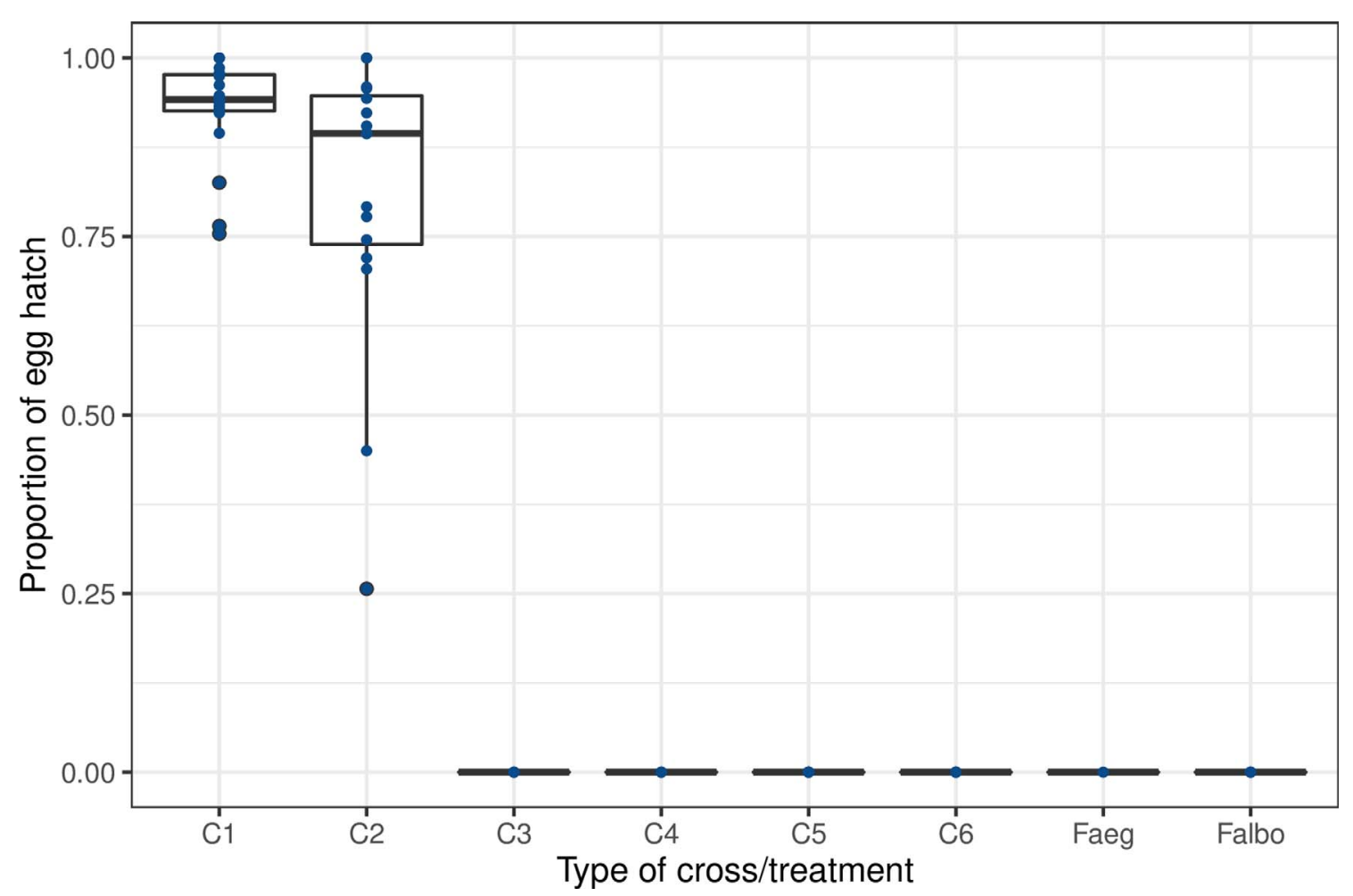

Figure 2. Egg hatch in sympatric crosses between Ae. aegypti and Ae. albopictus strains from La Réunion island. C denotes the cross and numbers (1-6) are related to the cross identity. $\mathrm{C} 1=$ control Ae. albopictus (non-irradiated), $\mathrm{C} 2=$ control Ae. aegypti (non-irradiated), $\mathrm{C} 3=$ male Ae. albopictus (non-irradiated) $\times$ female Ae. aegypti, $\mathrm{C} 4=$ male Ae. albopictus (irradiated) $\times$ female Ae. aegypti, $\mathrm{C} 5=$ male Ae. aegypti (non-irradiated) $\times$ female Ae. albopictus, $\mathrm{C} 4=$ male Ae. aegypti (irradiated) $\times$ female Ae. albopictus.

Table 2. Comparison of survival rate between female Ae. aegypti in the cross-mating between sympatric Ae. aegypti and Ae. albopictus strains from La Réunion island.

\begin{tabular}{lcccc}
\hline & Estimate & Std. error & $z$ value & $p(>|z|)$ \\
\hline (Intercept) & $5.93 \mathrm{E}-16$ & $1.63 \mathrm{E}-01$ & 0 & 1 \\
crossctrlalbo & $-2.96 \mathrm{E}-01$ & $2.32 \mathrm{E}-01$ & -1.272 & 0.2032 \\
crossMaegFalbo & $5.04 \mathrm{E}-01$ & $2.02 \mathrm{E}-01$ & 2.492 & $0.0127^{*}$ \\
crossMalboFaeg & $-1.05 \mathrm{E}+00$ & $2.10 \mathrm{E}-01$ & -4.987 & $6.13 \mathrm{E}-07^{* * *}$ \\
\hline
\end{tabular}

Std.: standard. Ctrlalbo stands for control Ae. albopictus cross; MaegFalbo $=$ male Ae. aegypti $\times$ female Ae. albopictus; MalboFaeg $=$ male Ae. albopictus $\times$ female Ae. aegypti. ${ }^{*} p<0.05$ and $* * * p<0.001$.

on islands [7]. In contrast, in our study even an increase in ratios in favor of male Ae. albopictus did not significantly increase insemination of female Ae. aegypti. One study has pointed to the implication of population density on mating behavior [42], and found that male density significantly increased swarming behavior, number of mating pairs, and egg production of hetero-specific females, but not female insemination. They also found that Ae. aegypti males mate more readily with hetero-specific females than do Ae. albopictus males, and so if Ae. aegypti were released into the field they may mate with both Ae. aegypti and Ae. albopictus females, and reduce populations of both species by preventing offspring. There is no evidence that this would be the case on La Réunion island since we observed low reproductive success when crosses were performed in both directions. In addition, we observed that interspecific mating was detrimental to female Ae. aegypti survival. It has been previously documented that interspecific copulation and hybridization can reduce female reproductive success, but interspecific sexual harassment, which does not always result in interspecific copulation, can also adversely affect individual reproductive success and longevity by suppressing both sugar and blood feeding [47]. In white butterflies (Leptidea spp.), for example, the prolonged mating ritual of hetero-specific males decreases the number of opportunities that females have to mate with conspecific males even when interspecific copulation does not take place [24]. Similarly, in bean weevils (Callosobruchus spp.), males persistently chase hetero-specific females, causing reductions in the oviposition rate and shortened longevity of these females [34].

Ae. aegypti females pre-exposed to male Ae. albopictus were able to be inseminated by their conspecific male Ae. aegypti demonstrating that the La Réunion island Ae. aegypti strain has developed a resistance to satyrization. Carrasquilla and Lounibos [15] have shown that Ae. aegypti females, previously exposed to Ae. albopictus males, were rendered refractory to subsequent conspecific mating even when their spermathecae contained no hetero-specific sperm. Additional experiments demonstrated transfer of labelled semen from Ae. albopictus males to Ae. aegypti females and low production of viable eggs of females housed with conspecific males, following exposure to Ae. albopictus males, and 


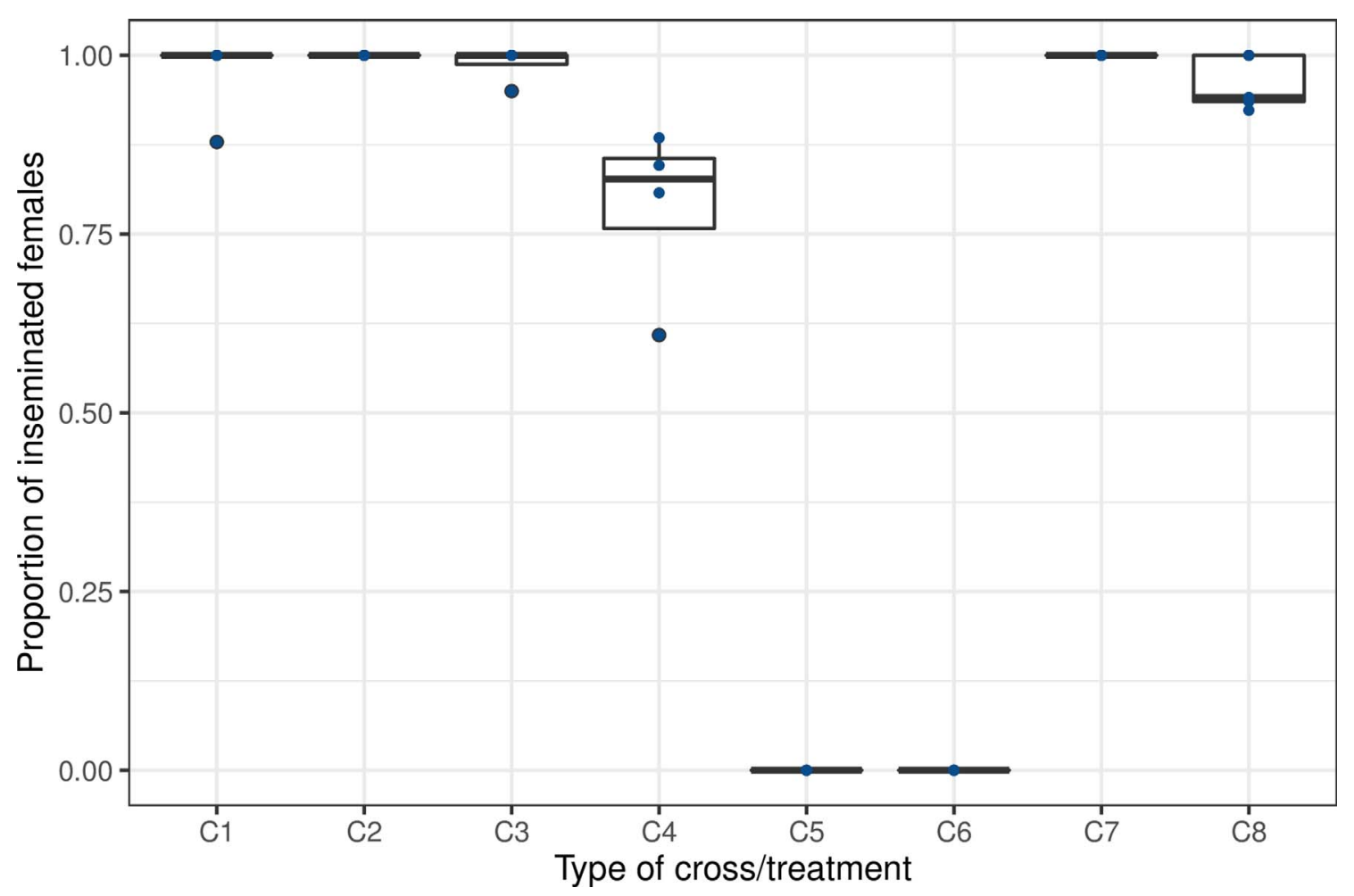

Figure 3. Pre-exposure effect on mating success. $\mathrm{C}$ denotes the cross, and numbers (1-8) are related to the cross identity. $\mathrm{C} 1=\mathrm{control}$ Ae. aegypti (non-irradiated), $\mathrm{C} 2=\mathrm{control}$ Ae. aegypti (irradiated), $\mathrm{C} 3=\mathrm{control}$ Ae. albopictus (non-irradiated), $\mathrm{C} 4=\mathrm{control}$ Ae. albopictus (irradiated), $\mathrm{C} 5=$ male Ae. albopictus (non-irradiated) $\times$ female Ae. aegypti, $\mathrm{C} 6=$ male Ae. albopictus (irradiated) $\times$ female Ae. aegypti, $\mathrm{C} 7=$ male Ae. aegypti (non-irradiated) $\times$ female Ae. aegypti (non-exposed to male Ae. albopictus), C8 = male Ae. aegypti (nonirradiated) $\times$ female Ae. aegypti (pre-exposed to male Ae. albopictus).

Table 3. Mean insemination and egg hatch rates $( \pm \mathrm{SE})$ in crosses between female Ae. aegypti (La Réunion island strain) and Ae. albopictus males from China, Italy (allopatric), and La Réunion island (sympatric).

\begin{tabular}{|c|c|c|c|c|c|}
\hline \multirow[t]{2}{*}{ Mating type } & \multicolumn{2}{|c|}{ Male } & \multirow[t]{2}{*}{ Female } & \multirow[t]{2}{*}{ Insemination rates $(\%)$} & \multirow[t]{2}{*}{ Hatch rates $(\%$} \\
\hline & Irradiated & Non-irradiated & & & \\
\hline \multirow[t]{2}{*}{ Interspecific } & China & & Réunion & $0.92 \pm 0.92^{\mathrm{a}}(95)$ & NA \\
\hline & & China & Réunion & $0 \pm 0^{\mathrm{a}}(82)$ & $0 \pm 0^{\mathrm{a}}$ \\
\hline \multirow[t]{2}{*}{ Conspecific } & China & & China & $100 \pm 0^{\mathrm{b}}(24)$ & NA \\
\hline & & China & China & $100 \pm 0^{\mathrm{b}}(48)$ & $100 \pm 0^{\mathrm{b}}$ \\
\hline \multirow[t]{2}{*}{ Interspecific } & Réunion & & Réunion & $1.92 \pm 1.11^{\mathrm{a}}(94)$ & NA \\
\hline & & Réunion & Réunion & $1.33 \pm 1.33^{\mathrm{a}}(74)$ & $0 \pm 0^{\mathrm{a}}$ \\
\hline \multirow{2}{*}{ Conspecific } & Réunion & & Réunion & $100 \pm 0^{b}$ & NA \\
\hline & & Réunion & Réunion & $100 \pm 0^{\mathrm{b}}(54)$ & $71.8 \pm 2.7^{\mathrm{c}}$ \\
\hline \multirow[t]{2}{*}{ Interspecific } & Italy & & Réunion & $0 \pm 0^{\mathrm{a}}(99)$ & NA \\
\hline & & Italy & Réunion & $5.5 \pm 3.68^{\mathrm{a}}(77)$ & $0 \pm 0^{\mathrm{a}}$ \\
\hline \multirow[t]{2}{*}{ Conspecific } & Italy & & Italy & $100 \pm 0^{\mathrm{b}}(50)$ & NA \\
\hline & & Italy & Italy & $100 \pm 0^{\mathrm{b}}(57)$ & $83.5 \pm 1.55^{\mathrm{c}}$ \\
\hline \multirow[t]{2}{*}{ Conspecific } & Réunion* & & Réunion & $100 \pm 0^{\mathrm{b}}(72)$ & NA \\
\hline & & Réunion* & Réunion & $100 \pm 0^{\mathrm{b}}(54)$ & $89.8 \pm 2.11^{\mathrm{c}}$ \\
\hline
\end{tabular}

"Interspecific" stands for crosses between female Ae. aegypti, La Réunion island strain and Ae. albopictus (strains from China, Italy, La Réunion island) and "conspecific" for control mating between male and female of the same species. Réunion*= male Ae. aegypti from La Réunion island. Numbers in parentheses represent the number of dissected females for insemination assessment. Different letters show significant differences between groups.

confirmed higher incidence of satyrization than expected, based on hetero-specific insemination rates. We did not observe this result after pre-exposing Ae. aegypti females to Ae. albopictus for seven days before replacing male Ae. albopictus by male
Ae. aegypti mosquitoes. It has been shown that interspecific pairs needed more time together before mating occurred. Bargielowki et al. [7] found that when female Ae. aegypti were exposed for up to three weeks, interspecific insemination 
increased significantly from $1 \%$ after one day, to $10 \%$ after one week, and to more than $50 \%$ after three weeks. However, assuming that most released sterile males will survive around one week in the field, it is unlikely that most released males would be able to find and mate with females after three weeks in the wild $[9,30]$. These results indicate that in areas where Ae. aegypti and Ae. albopictus co-occur, releasing sterile male Ae. albopictus may not suppress Ae. aegypti populations. It would be more beneficial to suppress the species with the smallest population, before further planning to control the second species, assuming the epidemiological impact of each species was equal.

In our study, female Ae. aegypti pre-exposed to male Ae. albopictus produced eggs which had similar egg hatch when mated with their conspecifics, meaning that females had not been inseminated by the Ae. albopictus males. The females that later mated with their conspecifics and laid eggs were apparently fully fertilized by conspecific sperm. However, it has been shown that the satyrization effect could be underestimated when evaluation of mating status of females is based on whether the spermathecae were filled with sperm or not [15]. It therefore cannot be ruled out that some females might have been inseminated when pre-exposed to Ae. albopictus based on the variation observed in egg hatch. Bargielowski et al. [7] demonstrated that multiple inseminations can occur in older female Ae. aegypti when the effects of accessory gland proteins have worn off, and in females mated to sperm-depleted males. In any case, hetero-specific sperm is known to be stored in separate spermathecae [7] and so was presumably not significantly used for egg fertilization.

Allopatric Ae. albopictus males did not perform better than sympatric males and anti-satyrization effects seem to protect against allopatric populations. This shows that resistance to one strain confers resistance to others. Honórios et al. [28] demonstrated that only some populations of Ae. albopictus are capable of satyrization. Female Ae. aegypti from populations allopatric to Ae. albopictus in the field were more susceptible to interspecific mating than females from sympatric populations, and selection experiments in cages confirmed the rapid development of resistance to satyrization in the laboratory, as well as changes in behavior toward conspecifics associated with increased satyrization resistance [5]. The fact that the Ae. aegypti populations persist in La Réunion island ravines as opposed to urban environments could be due to some genetic differentiation from domestic subspecies. Lounibos and Juliano [39] have recently pointed out that the feral subspecies Ae. aegypti formosus is expected to behave differently than the domestic subspecies but populations of this species from Madagascar, La Réunion island and Mayotte have not been tested yet for genetic distinctiveness from Ae. aegypti (aegypti) to the best of our knowledge. In any case, a signature of selection in the Ae. aegypti genome to a specific type of interspecific interaction (mating) was found by Burford Reiskind et al. [13] allowing the identification of its genetic basis.

When considering a regional approach for Aedes control using the SIT, compatibility of strains as well as species may be important as it would allow strains to be imported for release from nearby countries where they can be more easily reared and/or irradiated. Damiens et al. [18] demonstrated that male
Ae. albopictus from Mauritius and Seychelles islands, about 50-200 km away from La Réunion island, were compatible and could successfully inseminate female Ae. albopictus regardless of their origin. A regional SIT mass-rearing program could therefore be considered, with a good transportation method, but the release of sterile Ae. albopictus males may not have the added benefit of satyrizing the local Ae. aegypti population if an overflooding of irradiated male Ae. albopictus leads to similar results.

The development of resistance to satyrization in the Ae. aegypti strain shows that strong competition between the sympatric Ae. aegypti and Ae. albopictus probably occurs on La Réunion island. An SIT project against Ae. albopictus would not have an effect on Ae. aegypti populations, and other mechanisms such as larval competition probably explain the current geographical retraction of Ae. aegypti to the ravines. Bagny et al. [4] observed that this narrow distribution of Ae. aegypti was due to its poorer ability to cope with unfavorable temperatures and to its lower competition between larvae for resources compared to Ae. albopictus [31]. The two species may co-exist as long as the dominant Ae. albopictus is present and the resistance could be maintained by satyrization pressure [8]. Global climate change may favor an increase in the population size of Ae. aegypti [32], which is a greater vector of arboviral diseases including dengue, chikungunya, yellow fever, and Zika. Therefore, suppressing or eliminating Ae. albopictus will likely promote expansion of Ae. aegypti [26]. Whilst it may be important to target the most epidemiologically important vector first [1], considering its limited distribution, the eradication of the Ae. aegypti population may be seen as a first priority.

Acknowledgements. Authors are very grateful to the Institute of Research for Development (IRD) in La Réunion island, Wolbaki, and CAA for providing to the IPCL the Ae. aegypti and Ae. albopictus strains (La Réunion island), the Ae. albopictus strain (China), and the Ae. albopictus strain (Italy), respectively. The authors thank Adel Ali for technical assistance.

\section{Author contributions}

HM carried out the lab experiments, data analysis, and contributed to the design of the study and drafting of the manuscript. JG, RL, and HY contributed to the design of the study and drafting of the manuscript. JB helped in data analysis and drafting of the manuscript. JG and JB supervised this work. HY, RL, JG, and JB critically revised the manuscript. All authors gave final approval for publication.

\section{Competing interests}

The authors declare that they have no competing interests.

\section{Data availability}

All data generated or analyzed during this study are included in this published article. 


\section{Funding}

This study was supported by the Joint Food and Agricultural Organization of the United Nations/International Atomic Energy Agency, Division of Nuclear Techniques in Food and Agriculture and by the United States of America State Department through the grant entitled "Surge expansion for the sterile insect technique to control mosquito populations that transmit the Zika virus". Funders had no role in the study design, content of the article, or the decision to publish.

\section{References}

1. Alphey L, Benedict M, Bellini R, Clark GG, Dame DA, Service MW, Dobson SL. 2010. Sterile-insect methods for control of mosquito-borne diseases: an analysis. Vector Borne and Zoonotic Diseases, 10, 295-311.

2. Arrêté No 2019-2213. Autorisant l'IRD à procéder à des lâchers de moustiques stériles en vue d'études entomologiques au sein du quartier Duparc à Sainte-Marie - 13/06/2019.

3. Bagny L, Delatte H, Quilici S, Fontenille D. 2009. Progressive decrease in Aedes aegypti distribution in Reunion Island since the 1900s. Journal of Medical Entomology, 46, 1541-1545.

4. Bagny Beilhe L, Delatte H, Juliano SA, Fontenille D, Quilici S. 2013. Ecological interactions in Aedes species on Reunion Island. Medical and Veterinary Entomology, 27, 387-397.

5. Bargielowski I, Lounibos LP. 2014. Rapid evolution of reduced receptivity to interspecific mating in the dengue vector Aedes aegypti in response to satyrization by invasive Aedes albopictus. Evolutionary Ecology, 28, 193-203.

6. Bargielowski IE, Lounibos LP, Carrasquilla MC. 2013. Evolution of resistance to satyrization through reproductive character displacement in populations of invasive dengue vectors. Proceedings of the National Academy of Sciences of the United States of America, 110, 2888-2892.

7. Bargielowski IE, Blosser E, Lounibos LP. 2015. The effects of interspecific courtship on the mating success of Aedes aegypti and Aedes albopictus (Diptera: Culicidae) males. Annals of the Entomological Society of America, 108, 513-518.

8. Bargielowski I, Honório NA, Blosser EM, Lounibos LP. 2019. Rapid loss of resistance to satyrization in invasive mosquitoes and the effects of age on interspecific mating frequency. Journal of Medical Entomology, 56, 329-333.

9. Bellini R, Albieri A, Balestrino F, Carrieri M, Porretta D, Urbanelli S, Calvitti M, Riccardo MR, Maini S. 2010. Dispersal and survival of Aedes albopictus (Diptera: Culicidae) males in Italian urban areas and significance for sterile insect technique application. Journal of Medical Entomology, 7, 1082-1091.

10. Benedict MQ, Levine RS, Hawley WA, Lounibos LP. 2007. Spread of the tiger: global risk of invasion by the mosquito Aedes albopictus. Vector Borne and Zoonotic Diseases, 7, 76-85.

11. Bhatt S, Gething PW, Brady OJ, Messina JP, Farlow AW, Moyes CL, Drake JM, Brownstein JS, Hoen AG, Sankoh O, Myers MF, George DB, Jaenisch T, Wint GRW, Simmons CP, Scott TW, Farrar JJ, Hay SI. 2013. The global distribution and burden of dengue. Nature, 496, 504-507.

12. Bouyer J, Yamada H, Pereira R, Bourtzis K, Vreysen MJB. 2020. Phased conditional approach for mosquito management using sterile insect technique. Trends in Parasitology, S14714922(20), 30014-3.

13. Burford Reiskind MO, Labadie P, Bargielowski I, Lounibos LP, Reiskind MH. 2018. Rapid evolution and the genomic consequences of selection against interspecific mating. Molecular Ecology, 27, 3641-3654.

14. Burnham KP, Anderson DR. 2003. Model selection and multimodel inference: a practical information-theoretic approach. Berlin: Springer Science \& Business Media.

15. Carrasquilla MC, Lounibos LP. 2015. Satyrization without evidence of successful insemination from interspecific mating between invasive mosquitoes. Biology Letters, 11, 20150527.

16. Chouin-Carneiro T, Vega-Rua A, Vazeille M, Yebakima A, Girod R, Goindin D, Dupont-Rouzeyrol M, Lourençode-Oliveira R, Failloux AB. 2016. Differential Susceptibilities of Aedes aegypti and Aedes albopictus from the Americas to Zika Virus. PLoS Neglected Tropical Diseases, 10, e0004543.

17. Conseil scientifique de l'Agence française pour la biodiversité Séance des 26-27 avril 2018 - Délibération ${ }^{\circ}$ CS/2018-01.

18. Damiens D, Lebon C, Wilkinson DA, Dijoux-Millet D, Le Goff G, Bheecarry A, Gouagna LC. 2016. Cross-mating compatibility and competitiveness among Aedes albopictus strains from distinct geographic origins - implications for future application of SIT Programs in the South West Indian Ocean Islands. PLoS One, 11, e0163788.

19. de Araújo TVB, Rodrigues LC, de Alencar Ximenes RA, de Barros Miranda-Filho D, Montarroyos UR, de Melo APL, Valongueiro S, de Albuquerque MFPM, Souza WV, Braga C, Filho SPB, Cordeiro MT, Vazquez E, Di Cavalcanti Souza Cruz D, Henriques CMP, Bezerra LCA, da Silva Castanha PM, Dhalia R, Marques-Júnior ETA, Martelli CMT, Investigators from the Microcephaly Epidemic Research Group, Brazilian Ministry of Health, Pan American Health Organization, Instituto de Medicina Integral Professor Fernando Figueira, State Health Department of Pernambuco. 2016. Association between Zika virus infection and microcephaly in Brazil, January to May, 2016: preliminary report of a case-control study. Lancet Infectious Diseases, 16, 1356-1363.

20. Delatte H, Dehecq JS, Thiria J, Domerg C, Paupy C, Fontenille D. 2008. Geographic distribution and developmental sites of Aedes albopictus (Diptera: Culicidae) during a Chikungunya epidemic event. Vector Borne and Zoonotic Diseases, 8, 25-34.

21. Dyck VA, Hendrichs JP, Robinson AS. 2005. The sterile insect technique: principles and practice in area-wide integrated pest management. Dordrecht: Springer.

22. FAO/IAEA. 2017. Guidelines for routine colony maintenance of Aedes mosquito species - version 1.0 [Internet]. p. 18. Available from: https://www.iaea.org/resources/manual/guidelines-for-routine-colony-maintenance-of-aedes-mosquito-speciesversion-10.

23. Focks DA. 1980. An improved separator for the developmental stages, sexes, and species of mosquitoes (Diptera: Culicidae). Journal of Medical Entomology, 17, 567-568.

24. Friberg M, Leimar O, Wiklund C. 2013. Heterospecific courtship, minority effects and niche separation between cryptic butterfly species. Journal of Evolutionary Biology, 26, 971-979.

25. Giatropoulos A, Papachristos DP, Koliopoulos G, Michaelakis A, Emmanouel N. 2015. Asymmetric mating interference between two related mosquito species: Aedes (Stegomyia) albopictus and Aedes (Stegomyia) cretinus. PLoS One, 10, e0127762.

26. HCB Scientific Committee. 2017. Scientific Opinion of the High Council for Biotechnology concerning use of genetically modified mosquitoes for vector control in response to the referral of 12 October 2015 (Ref. HCB-2017.06.07). Paris: HCB. p. 142. Available online: http://www.hautconseildesbiotechnologies.fr.

27. Honma A, Kumano N, Noriyuki S. 2019. Killing two bugs with one stone: a perspective for targeting multiple pest species by incorporating reproductive interference into sterile insect technique. Pest Management Science, 75, 571-577. 
28. Honório NA, Carrasquilla MC, Bargielowski I, Nishimura N, Swan T, Lounibos LP. 2018. Male origin determines satyrization potential of Aedes aegypti by invasive Aedes albopictus. Biological Invasions, 20, 653-664.

29. Hurvich CM, Tsai CL. 1995. Model selection for extended quasi-likelihood models in small samples. Biometrics, 51, 1077-1084.

30. Iyaloo DP, Damiens D, Sunita F, Elahee KB, Bheecarry A. 2019. Dispersal and survival of radio-sterilised male Aedes albopictus Skuse (Diptera: Culicidae) and estimation of the wild populations in view of an sterile insect technique programme in Pointe des Lascars, Mauritius. International Journal of Tropical Insect Science, 39, 63.

31. Juliano SA. 2009. Species interactions among larval mosquitoes: context dependence across habitat gradients. Annual Review of Entomology, 54, 37-56.

32. Juliano SA, Lounibos LP, O'Meara GF. 2004. A field test for competitive effects of Aedes albopictus on Aedes aegypti in South Florida: differences between sites of coexistence and exclusion? Oecologia, 139, 583-593.

33. Kaplan L, Kendell D, Robertson D, Livdahl T, Khatchikian C. 2010. Aedes aegypti and Aedes albopictus in Bermuda: extinction, invasion, invasion and extinction. Biological Invasions, 12, 3277-3288.

34. Kishi S, Nishida T, Tsubaki Y. 2009. Reproductive interference determines persistence and exclusion in species interactions. Journal of Animal Ecology, 78, 1043-1049.

35. Knipling EF. 1959. Sterile-male method of population control: successful with some insects, the method may also be effective when applied to other noxious animals. Science, 130, 902-904.

36. Kyle JL, Harris E. 2008. Global spread and persistence of dengue. Annual Review of Microbiology, 62, 71-92.

37. Levy-Blitchtein S, Del Valle-Mendoza J. 2016. Zika virus is arriving at the American continent. Asian Pacific Journal of Tropical Medicine, 9, 1019-1021.

38. Lounibos LP. 2002. Invasions by insect vectors of human disease. Annual Review of Entomology, 47, 233-266.

39. Lounibos LP, Juliano SA. 2018. Where Vectors Collide: the importance of mechanisms shaping the realized niche for modeling ranges of invasive Aedes Mosquitoes. Biological Invasions, 20, 1913-1929.

40. Lounibos LP, Bargielowski I, Carrasquilla MC, Nishimura N. 2016. Coexistence of Aedes aegypti and Aedes albopictus
(Diptera: Culicidae) in Peninsular Florida two decades after competitive displacements. Journal of Medical Entomology, 53, 1385-1390.

41. Maïga H, Gilles JRL, Lees RS, Yamada H, Bouyer J. 2020. Demonstration of resistance to satyrization behavior in Aedes aegypti (Linnaeus) from La Réunion island. bioRxiv 2020.02.10.942839. https://doi.og/10.1101/2020.02.10.942839.

42. Marcela P, Abu Hassan A, Hamdan A, Dieng H, Kumara TK. 2015. Interspecific cross-mating between Aedes aegypti and Aedes albopictus laboratory strains: implication of population density on mating behaviors. Journal of the American Mosquito Control Association, 31, 313-320.

43. Nasci RS, Hare SG, Willis FS. 1989. Interspecific mating between Louisiana strains of Aedes albopictus and Aedes aegypti in the field and laboratory. Journal of the American Mosquito Control Association, 5, 416-421.

44. Paupy C, Ollomo B, Kamgang B, Moutailler S, Rousset D, Demanou M, Hervé JP, Leroy E, Simard F. 2010. Comparative role of Aedes albopictus and Aedes aegypti in the emergence of Dengue and Chikungunya in central Africa. Vector Borne and Zoonotic Diseases, 10, 259-266.

45. Ribeiro JMC. 1988. Can satyrs control pests and vectors? Journal of Medical Entomology, 25, 431-440.

46. RStudio Team. 2016. RStudio: Integrated Development for R. Boston, MA: RStudio Inc. URL: http://www.rstudio.com/.

47. Soghigian J, Gibbs K, Stanton A, Kaiser R, Livdahl T. 2014. Sexual harassment and feeding inhibition between two invasive dengue vectors. Environmental Health Insights, 8, 61-66.

48. Tripet F, Lounibos LP, Robbins D, Moran J, Nishimura N, Blosser EM. 2011. Competitive reduction by satyrization? Evidence for interspecific mating in nature and asymmetric reproductive competition between invasive mosquito vectors. Journal of the American Mosquito Control Association, 85, 265-270.

49. World Health Organization. 2017. Global vector control response 2017-2030.

50. World Health Organization. 2019. Dengue fever - Réunion, France. https://www.who.int/csr/don/01-may-2018-dengue-reunion/ en/ (Assessed on 11 Sep 2019).

51. Zheng ML, Zhang DJ, Damiens DD, Lees RS, Gilles JR. 2015. Standard operating procedures for standardized mass rearing of the dengue and chikungunya vectors Aedes aegypti and Aedes albopictus (Diptera: Culicidae). II. Egg storage and hatching. Parasites \& Vectors, 8, 348.

Cite this article as: Maïga H, Gilles JRL, Susan Lees R, Yamada H \& Bouyer J. 2020. Demonstration of resistance to satyrization behavior in Aedes aegypti from La Réunion island. Parasite 27, 22. on all aspects of human and animal parasitology

Reviews, articles and short notes may be submitted. Fields include, but are not limited to: general, medical and veterinary parasitology; morphology, including ultrastructure; parasite systematics, including entomology, acarology, helminthology and protistology, and molecular analyses; molecular biology and biochemistry; immunology of parasitic diseases; host-parasite relationships; ecology and life history of parasites; epidemiology; therapeutics; new diagnostic tools.

All papers in Parasite are published in English. Manuscripts should have a broad interest and must not have been published or submitted elsewhere. No limit is imposed on the length of manuscripts.

Parasite (open-access) continues Parasite (print and online editions, 1994-2012) and Annales de Parasitologie Humaine et Comparée (1923-1993) and is the official journal of the Société Française de Parasitologie. 